\title{
Intravenous acetaminophen analgesia after cardiac surgery: A randomized, blinded, controlled superiority trial
}

\author{
Negmeldeen F. Mamoun, MD, PhD, ${ }^{\mathrm{a}, \mathrm{b}}$ Peirong Lin, MD, ${ }^{\mathrm{b}}$ Nicole M. Zimmerman, MS, ${ }^{\mathrm{b}, \mathrm{c}}$ \\ Edward J. Mascha, PhD, ${ }^{\mathrm{b}, \mathrm{c}}$ Stephanie L. Mick, MD, ${ }^{\mathrm{d}}$ Steven R. Insler, DO, ${ }^{\mathrm{a}, \mathrm{b}}$ Daniel I. Sessler, MD, ${ }^{\mathrm{b}}$ and \\ Andra E. Duncan, MD ${ }^{\mathrm{a}, \mathrm{b}}$
}

\begin{abstract}
Background: Pain after cardiac surgery traditionally has been controlled by intravenous opioids and nonsteroidal antiinflammatory drugs. An intravenous analgesic with fewer adverse effects is needed. Therefore, we tested the primary hypothesis that intravenous acetaminophen is more effective than placebo for pain management, which was defined a priori as superior on either pain intensity score and/or opioid consumption and not worse on either.
\end{abstract}

Methods: In this single-center, double-blind trial, 147 patients having cardiac surgery via median sternotomy were randomized to receive either $1 \mathrm{~g}$ of intravenous acetaminophen ( 73 patients) every 6 hours for 24 hours or comparable placebo (74 patients) starting in the operating room after sternal closure. Cumulative opioid consumption (in morphine equivalents) and pain intensity scores (on a 0-10 Numeric Rating Scale) were measured at 4, 6, 8, 12, 16, 20, and 24 hours after surgery. We estimated ratio of mean opioid consumption by using multivariable linear regression (noninferiority delta $=1.15$ ) and pain score difference by using repeated measures regression (noninferiority delta $=1$ ).

Results: Acetaminophen was superior to placebo on mean pain intensity scores and noninferior on opioid consumption, with estimated difference in mean pain ( $95 \%$ confidence interval) of $-0.90(-1.39,-0.42), P<.001$ (superior), and estimated ratio of means in opioid consumption ( $90 \%$ confidence interval) of 0.89 (0.73-1.10), $P=.28$ (noninferior; not superior).

Conclusions: Intravenous acetaminophen reduced pain after cardiac surgery, but not opioid consumption. Intravenous acetaminophen can be an effective analgesic adjunct in patients recovering from median sternotomy. ( $\mathrm{J}$ Thorac Cardiovasc Surg 2016;152:881-9)

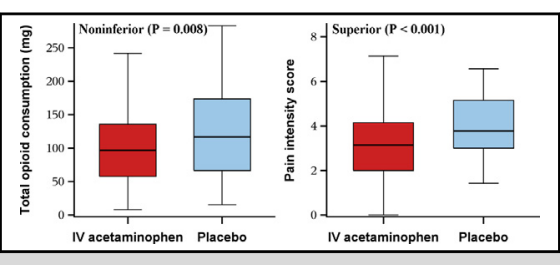

IV acetaminophen was noninferior on opioid consumption and superior on pain scores.

Central Message

Intravenous acetaminophen significantly reduced pain, but not opioid consumption, after cardiac surgery done via median sternotomy.

\section{Perspective}

Pain after cardiac surgery traditionally has been controlled by intravenous opioids and nonsteroidal antiinflammatory drugs. Intravenous acetaminophen has fewer adverse effects and was found to significantly reduce pain intensity scores, but not opioid consumption, after cardiac surgery. Intravenous acetaminophen may be an effective component of a multimodal analgesic strategy after median sternotomy.

See Editorial Commentary page 890.
Multimodal analgesic strategies depend on the synergistic effects of various classes of analgesics. Combining several agents thus permits reductions in individual drug doses and consequent adverse effects. The World Health

\footnotetext{
From the Departments of ${ }^{a}$ Cardiothoracic Anesthesia and ${ }^{b}$ Outcomes Research, Anesthesiology Institute, and Departments of ${ }^{\mathrm{c}}$ Quantitative Health Sciences and ${ }^{\mathrm{d}}$ Cardiothoracic Surgery, Cleveland Clinic, Cleveland, Ohio.

This study was supported by departmental and institutional funds and a grant from Cadence Pharmaceuticals (grant No. CADPH1303NM), which was purchased subsequently by Mallinckrodt. The sponsors of the study did not participate in the design of the study, collecting, analyzing and interpreting the data, writing the report, or deciding to submit the report for publication.

The authors attest to having full freedom to explore the data and analyze the results. The authors had sole authority to make the final decision to submit the material for publication. The authors had no potential conflicts of interest and no financial interests with any commercial entity that would be affected by the publication.

The study had complex statistical methodology; therefore, 2 biostatisticians were needed to assist with the statistical design and analysis and were both included as authors.
}

Organization's Pain Ladder recommends administering nonopioid analgesics before adding opioids if warranted by the intensity of postoperative pain. ${ }^{1}$ Similarly, the American Society of Anesthesiologists recommends stepwise

Current affiliation of Dr Lin: Department of Anesthesiology, Beijing Anzhen Hospital.

This report describes a prospective randomized clinical trial. The author states that the report includes every item in the CONSORT checklist for a prospective randomized clinical trial.

The study was registered before patient enrolment at www.clinicaltrials.gov on March 28, 2013, registration number: NCT01822821

Received for publication Nov 21, 2015; revisions received March 14, 2016; accepted for publication April 23, 2016; available ahead of print May 26, 2016

Address for reprints: Negmeldeen F. Mamoun, MD, PhD, Departments of Cardiothoracic Anesthesia and Outcomes Research, Anesthesiology Institute, Cleveland Clinic, 9500 Euclid Ave, Cleveland, OH 44195 (E-mail: mamounn@ccf.org). 0022-5223/ $\$ 36.00$

Copyright $(C) 2016$ by The American Association for Thoracic Surgery http://dx.doi.org/10.1016/j.jtcvs.2016.04.078 

Abbreviations and Acronyms
ALT = alanine aminotransferase
ASD $=$ absolute standardized difference
AST $=$ aspartate aminotransferase
$\mathrm{CI}=$ confidence interval
$\mathrm{CV}=$ coefficient of variation
ICU = intensive care unit
IV = intravenous
LOS = length of stay
PCA = patient-controlled analgesia
PONV $=$ postoperative nausea and vomiting
RASS $=$ Richmond Agitation Sedation Scale
$\mathrm{SD}=$ standard deviation

Scanning this QR code will take you to the supplemental table for this article.

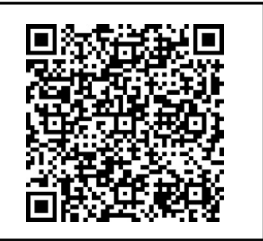

multimodal analgesic regimens for postoperative pain control via round-the-clock nonopioid analgesics as the initial treatment. $^{2}$

Oral acetaminophen usually is used as an initial treatment of acute pain because of its high therapeutic index. ${ }^{3}$ The Food and Drug Administration approved an intravenous (IV) formulation of the drug in 2010, which brought new potential to this century-old drug. Although IV acetaminophen might not offer a clear benefit over the oral formulation in patients who can tolerate oral intake, ${ }^{4}$ it may be more helpful in patients who remain intubated postoperatively or those who develop delayed gastric emptying or postoperative nausea and vomiting (PONV). Further, IV acetaminophen avoids variable first-pass elimination that accompanies oral administration, and thus has a faster onset ${ }^{5}$ and a greater peak plasma concentration. ${ }^{6}$ There is consequently greater cerebrospinal fluid penetration with the IV preparation, along with more predictable pharmacokinetic behavior and bioavailability.

Pain after cardiac surgery traditionally has been controlled with IV opioids and nonsteroidal antiinflammatory drugs, which have significant adverse effects. ${ }^{8}$ Therefore, there is an unmet need for a safer IV analgesic, such as IV acetaminophen. ${ }^{9}$ IV acetaminophen has been used effectively to control acute pain after various surgeries, ${ }^{10-14}$ but the extent to which it might help after cardiac surgery remains unclear. We therefore tested the primary hypothesis that IV acetaminophen is more effective than placebo for pain management after cardiac surgery. We defined a priori that acetaminophen would be considered more effective than placebo if it was superior on pain intensity score and/or opioid consumption and noninferior on both. We also tested the secondary hypotheses that IV acetaminophen reduces opioid-related adverse effects (PONV, sedation, and respiratory depression), reduces the duration of mechanical ventilation, and reduces intensive care unit (ICU) and hospital length of stay (LOS).

\section{METHODS}

This prospective, single-center, randomized, parallel-group, doubleblind trial was approved by the Cleveland Clinic Institutional Review Board and registered before patient enrollment at www.clinicaltrials.gov on March 28, 2013, registration number: NCT01822821, Principal investigator's name: Negmeldeen Mamoun. Written consent was obtained from each participating patient.

We screened adults 18 years of age or older who were scheduled for elective cardiac surgery performed via a median sternotomy at the Cleveland Clinic. Exclusion criteria included complex cardiac surgery such as multiple valve replacements or aortic arch surgery. Other exclusion criteria included previous cardiac surgery, moderate or severe right ventricular dysfunction, left ventricular dysfunction with ejection fraction $\leq 35 \%$, severe tricuspid regurgitation, severe lung disease requiring home oxygen therapy, preoperative renal insufficiency (creatinine $>2.0$ ) or hemodialysis, history of active liver disease or liver cirrhosis, chronic pain conditions that required daily preoperative opioid administration, pregnancy, weight less than $50 \mathrm{~kg}$, and allergy to fentanyl or acetaminophen.

\section{Protocol}

Patients were randomized (1:1) without stratification to IV acetaminophen or placebo. Allocations were concealed by a password-protected Web site. Randomization codes were computer-generated by using the PLAN procedure in SAS statistical software (SAS Institute, Cary, NC), using block randomization with a block size of either 2 or 4 patients. After enrollment, the Cleveland Clinic Investigational Drug Pharmacy blinded the designated study drug by repackaging acetaminophen or placebo (normal saline). Study drugs were labeled with codes that remained locked until completion of patient enrollment.

Anesthetic induction involved the administration of etomidate or propofol, fentanyl, midazolam, and a depolarizing or nondepolarizing muscle relaxant to facilitate intubation. Fentanyl, isoflurane, and nondepolarizing muscle relaxants were given for maintenance of anesthesia. Routine strategies for heparinization and initiation and separation from cardiopulmonary bypass were followed.

Four doses of IV acetaminophen $(1 \mathrm{~g}$ each) or an equal volume of identical-looking placebo were given over 15 minutes every 6 hours ( \pm 30 minutes) starting in the operating room after sternal closure, with subsequent doses given in the ICU. All patients were also offered patient-controlled analgesia (PCA). Fentanyl was the default drug (PCA settings: no basal rate, demand bolus dose of $20 \mu \mathrm{g}$, bolus interval every 6 minutes); however, hydromorphone was substituted (PCA settings: no basal rate, demand bolus dose of $0.2 \mathrm{mg}$, bolus interval every 6 minutes) if clinically indicated, that is, fentanyl was ineffective in decreasing pain intensity scores $<4 / 10$ on the Numeric Rating Scale, where 0 is no pain and 10 is the worst possible pain. ${ }^{15}$ Similarly, rescue analgesia included IV fentanyl or hydromorphone boluses, or oral oxycodone if PCA was ineffective in decreasing pain intensity scores $<4 / 10$. IV meperidine was given as needed for shivering. Wounds were not infiltrated with local anesthetics. No other form of acetaminophen was permitted, nor were topical lidocaine patches or nonsteroidal antiinflammatory drugs allowed. 


\section{Measurements}

All study data were collected by nurses and research personnel blinded to group allocation. Standard anesthesia care included use of routine monitors recommended by the American Society of Anesthesiologists, invasive arterial pressure, transesophageal echocardiography, and bladder temperature monitoring. Central venous pressures and, in selected cases, pulmonary artery pressures also were monitored.

Baseline patient characteristics were recorded, along with surgical details. Cumulative opioid consumption during the first 24 hours after surgery was calculated. Administered opioids were converted to morphine equivalents to account for all given opioids, where $10 \mathrm{mg}$ of IV morphine was equivalent to $100 \mu \mathrm{g}$ of IV fentanyl, $1.5 \mathrm{mg}$ of IV hydromorphone, $75 \mathrm{mg}$ of IV meperidine, and $20 \mathrm{mg}$ of oral oxycodone (Table E1). ${ }^{16}$ Pain intensity scores were evaluated at 4, 6, 8, 12, 16, 20, and 24 hours $( \pm 30$ minutes) after surgery.

The incidence of PONV was assessed and documented by ICU nurses during the first 24 hours after surgery. The Richmond Agitation Sedation Scale (RASS) score was evaluated at 8,16 , and 24 hours ( \pm 30 minutes) after surgery. ${ }^{17}$ The duration of mechanical ventilation, ICU LOS, and hospital LOS were recorded. Blood was sampled for alanine aminotransferase (ALT), aspartate aminotransferase (AST), and bilirubin on the first and second postoperative days.

\section{Statistical Analyses}

Analyses followed a modified intent-to-treat principle, which we defined a priori as including all randomized patients who received any study treatment (either IV acetaminophen or placebo). As planned a priori, we assessed balance of the randomized groups on potentially confounding baseline variables using absolute standardized difference (ASD), defined as the absolute difference in means or proportions divided by the pooled standard deviation (SD). Any variable with ASD $>0.20$ was considered imbalanced and was adjusted for in all analyses. ${ }^{18}$

Primary analyses. We assessed the effectiveness of IV acetaminophen (vs placebo) on pain management, measured by cumulative opioid consumption and pain intensity scores within the first 24 hours after surgery, using a joint hypothesis testing framework. ${ }^{19}$ IV acetaminophen was considered more effective than placebo if it was noninferior on both primary outcomes and superior on at least one. We thus planned to test for superiority only if IV acetaminophen was found to be noninferior on both outcomes. A priori, we defined noninferiority deltas as a ratio of means of 1.15 for opioid consumption (ie, no more than $15 \%$ greater in the acetaminophen group) and 1 point for Numeric Rating Scale pain intensity score (ie, no more than 1 point worse).

Joint hypothesis testing of pain score and opioid consumption was conducted at the overall 0.05 significance level, and all tests were 1-tailed in the direction favoring acetaminophen. No multiple testing adjustment was made when we tested for noninferiority because noninferiority was required on both outcomes (ie, 0.051 -tailed significance criterion, $90 \%$ confidence intervals [CIs]); however, we adjusted for multiple comparisons for superiority testing because superiority on either outcome (given noninferiority on both) was sufficient to reject the null hypothesis (ie, 0.0251 -tailed significance criterion, 95\% CI; Bonferroni correction).

Cumulative opioid consumption was normalized with a log transformation. For both noninferiority and superiority testing, we estimated the treatment effect on log opioid consumption by using a multivariable linear regression model and the treatment effect on pain intensity scores by using a repeated-measures linear regression model with an autoregressive correlation structure adjusting for time and testing the treatment-by-time interaction. Noninferiority was detected if the upper $90 \%$ confidence limit for treatment effect was less than the respective noninferiority delta. Superiority was found if the estimated upper $95 \%$ confidence limit fell below a ratio of means of 1 for opioid consumption and below 0 for pain score. Missing pain assessments were assumed to be missing at random.
Secondary analyses. We tested for superiority for all secondary outcomes by using 2-tailed tests. The effect of IV acetaminophen on PONV was estimated with a multivariable logistic regression model with a log link to estimate relative risk. We estimated the treatment effect on RASS scores at 8,16 , and 24 hours after surgery by using separate Wilcoxon rank sum tests with Hodges-Lehmann estimation of median difference for each time point. Missing RASS assessments were assumed to be missing at random.

The effect of IV acetaminophen on duration of mechanical ventilation, ICU LOS, and hospital LOS was assessed by the use of separate multivariable linear regression models. We estimated the treatment effect of IV acetaminophen on ALT, AST, and total bilirubin liver enzymes on the first and second postoperative days by using a repeated-measures linear regression model with an autoregressive correlation structure and adjusting for time and respective baseline preoperative liver enzyme levels. Time-to-event and enzyme outcomes were log-transformed to achieve normality.

We used an overall alpha of 0.05 for secondary analyses, testing each hypothesis at the 0.00625 significance level to control for multiple testing (ie, $0.05 / 8$, Bonferroni correction). Groups were compared on RASS scores at each of 3 time points using a 0.002 significance criterion (ie, $0.00625 / 3$ ). We completed the primary and secondary analyses using SAS 9.3 (SAS Institute) and R statistical software version 2.15.3 (R Project for Statistical Computing, Vienna, Austria).

Sample size and power. Opioid consumption. Sinatra et $\mathrm{al}^{10}$ evaluated the analgesic efficacy of acetaminophen after major orthopedic surgery, reporting the opioid consumption coefficient of variation (ie, $\mathrm{SD} /$ mean $\times 100$ ) of $91 \%$ in the placebo group. We assumed a slightly more conservative coefficient of variation of $75 \%$ and that the treatment effect favored acetaminophen by $30 \%$ (ie, true ratio of geometric means of 0.70 ), requiring 150 total patients ( 75 per group) at the 0.025 significance level ( 0.05 overall for 2 outcomes) to have $90 \%$ power with a 1 -tailed test to detect superiority of acetaminophen to placebo on opioid consumption. Pain intensity scores. Memis et a ${ }^{11}$ evaluated the analgesic efficacy of acetaminophen after major surgery and found an SD of 0.3 in the placebo group. We assumed an SD as high as 1.8 points in each group. To have $90 \%$ power at the 0.025 significance level (overall 0.05 with 2 outcomes) for detecting superiority of acetaminophen to placebo in a 1-tailed test with underlying difference of 1 point (favoring acetaminophen) and SD of 1.8 , we required 140 total patients.

We therefore enrolled 150 patients ( 75 per group) to have $90 \%$ power at the 0.05 significance level to reject the joint null hypothesis. We assumed an underlying superiority on both outcomes (at least 1 outcome needed to be superior to reject the null hypothesis), so sample size was largely driven by tests for superiority rather than noninferiority.

\section{RESULTS}

A total of 1845 patients were screened between May 2013 and December 2014, of whom 155 provided written consent. Five consented patients were excluded from the study before randomization because surgery was cancelled or surgical plan was changed; thus, 150 patients completed enrollment. Among the 150 patients enrolled in the trial (75 patients per group), 2 patients in the acetaminophen group and 1 patient in the placebo group withdrew after randomization but before receiving treatment and were thus excluded from analyses (Figure 1).

Table 1 presents patient baseline and demographic characteristics. Patients in the IV acetaminophen group were older and more likely to have diabetes mellitus on the basis of our a priori definition of imbalance 


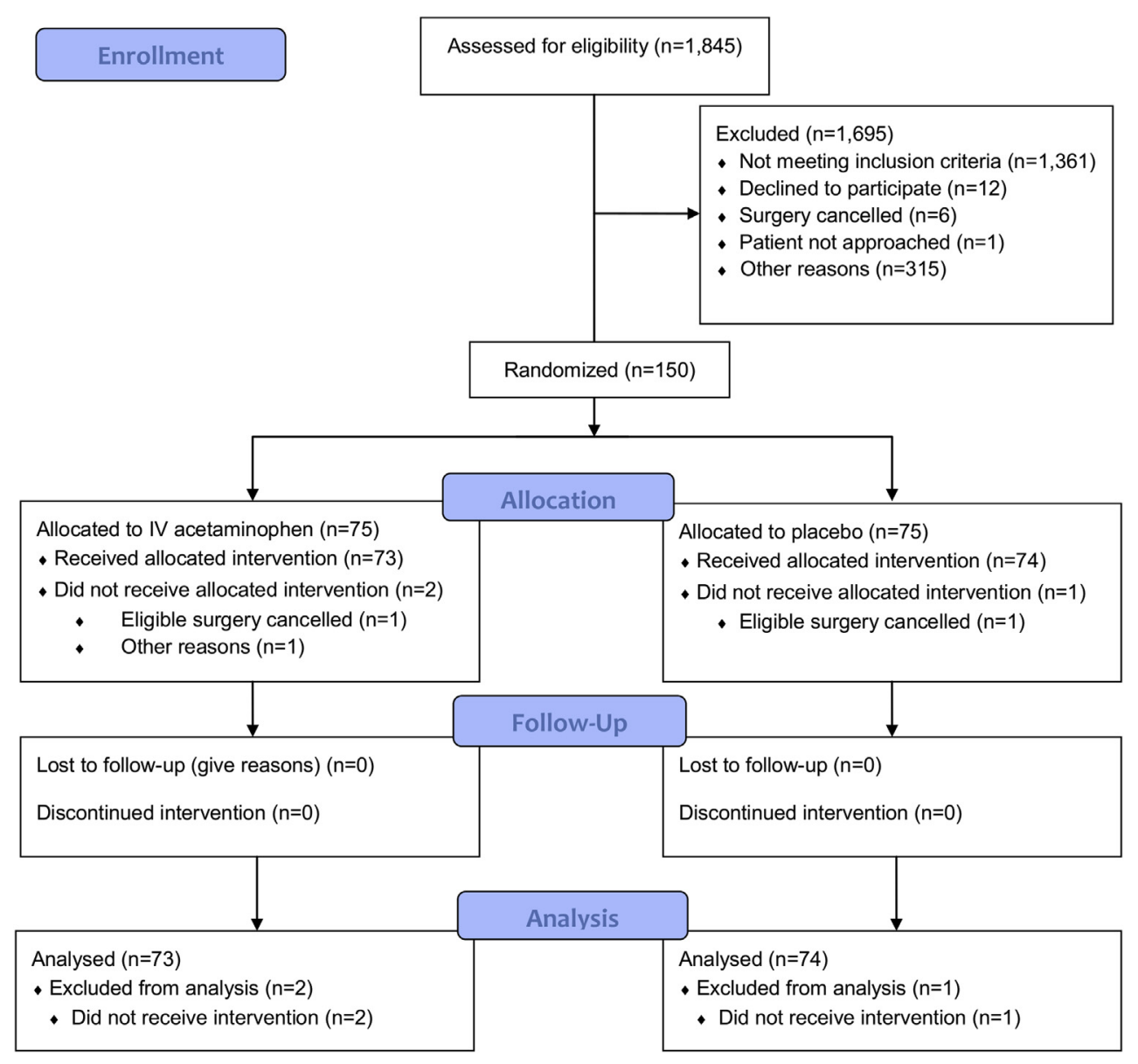

FIGURE 1. CONSORT (ie, CONsolidated Standards of Reporting Trials) flow diagram. $I V$, Intravenous.

(ie, ASD > 0.2), so all analyses were adjusted for age and diabetes status. Other baseline variables and preoperative characteristics were balanced between groups.

\section{Primary Analyses}

Summary. IV acetaminophen was found to be more effective than placebo on pain management because it was superior on pain intensity scores and noninferior on opioid consumption, with a difference in mean pain score $(95 \%$ CI) of $-0.90(-1.39,-0.42), P<.001$ (superior) and estimated ratio of means in opioid consumption $(90 \% \mathrm{CI})$ of 0.89 (0.73-1.10), $P=.28$ (noninferior; not superior) (Figure 2). Mean \pm SD postoperative pain scores (average for each patient) within the first 24 hours after surgery were $3.1 \pm 1.6$ for IV acetaminophen patients and $4.0 \pm 1.4$ for placebo (Table 2; Figure 3), and noninferior on cumulative opioid consumption (median [first quartile, third quartile] opioid dose in morphine equivalents was $97[58,136] \mathrm{mg}$ for IV acetaminophen and 117 [66, 174] $\mathrm{mg}$ for placebo; Table 2; Figure 4).

Noninferiority. The ratio of means $(90 \%$ CI) for IV acetaminophen versus placebo patients on cumulative opioid consumption was 0.89 (0.75-1.06). Because the upper confidence limit of the $90 \%$ CI was less than the specified delta of 1.15 , we conclude noninferiority of IV acetaminophen compared with placebo $(P=.008)$.

IV acetaminophen caused an estimated mean $(90 \% \mathrm{CI})$ reduction of $-0.90(-1.31,-0.50)$ in postoperative pain scores compared with placebo. We thus claimed noninferiority of IV acetaminophen to placebo because the upper limit of the $90 \%$ CI was less than the a priori noninferiority delta of 1 point $(P<.001)$. Mean pain intensity scores were greatest within a few hours after surgery and decreased over time. Treatment effect did not differ over time (treatment-by-time interaction $P=.35$ ).

Superiority. Because IV acetaminophen was noninferior on both opioid consumption and pain intensity scores, we proceeded to superiority testing (Figure 5). IV acetaminophen was not superior to placebo on mean opioid consumption, with an estimated ratio of means $(95 \% \mathrm{CI})$ of 0.89 ([0.73-1.10]; $P=.28$ ); however, IV acetaminophen was superior to placebo on pain intensity scores, with a mean $(95 \%$ CI $)$ change of $-0.90([-1.39,-0.42]$; $P<.001$, Figure 5). Therefore, the joint null hypothesis was rejected, and IV acetaminophen was found more effective on pain management than placebo. 
TABLE 1. Baseline and demographic characteristics of study population

\begin{tabular}{|c|c|c|c|}
\hline Factor & $\begin{array}{c}\text { Acet } \\
(\mathbf{n}=73)\end{array}$ & $\begin{array}{l}\text { Placebo } \\
(n=74)\end{array}$ & ASD* \\
\hline \multicolumn{4}{|l|}{ Demographics } \\
\hline Age, y & $62 \pm 14$ & $59 \pm 14$ & 0.22 \\
\hline Female, n $(\%)$ & $24(33)$ & $24(32)$ & 0.01 \\
\hline BMI, $\mathrm{kg} / \mathrm{m}^{2}$ & $30 \pm 6$ & $30 \pm 6$ & 0.08 \\
\hline \multicolumn{4}{|l|}{ Medical history } \\
\hline Hypertension, $\mathrm{n}(\%)$ & $47(64)$ & $47(64)$ & 0.02 \\
\hline Diabetes mellitus, n (\%) & $14(19)$ & $5(7)$ & 0.38 \\
\hline \multicolumn{4}{|l|}{ Procedure information } \\
\hline Valve surgery, $\mathrm{n}(\%)$ & $36(49)$ & $37(50)$ & 0.01 \\
\hline CABG, n $(\%)$ & $21(29)$ & $17(23)$ & 0.13 \\
\hline Myectomy, n (\%) & $24(33)$ & $18(24)$ & 0.19 \\
\hline $\begin{array}{l}\text { Ascending aortic replacement, } \\
\mathrm{n}(\%)\end{array}$ & $15(21)$ & $17(23)$ & 0.06 \\
\hline Other surgeries, $\nmid \mathrm{n}(\%)$ & $23(32)$ & $17(23)$ & 0.19 \\
\hline $\mathrm{CPB}, \mathrm{n}(\%)$ & $73(100)$ & $74(100)$ & $<0.001$ \\
\hline $\mathrm{CPB}$ duration, min & $73 \pm 36$ & $72 \pm 34$ & 0.01 \\
\hline Aortic cross-clamp, n (\%) & $73(100)$ & $74(100)$ & $<0.001$ \\
\hline Intraoperative fentanyl dose, $\mu \mathrm{g}$ & $1021 \pm 230$ & $1018 \pm 352$ & 0.01 \\
\hline \multicolumn{4}{|l|}{ Preoperative labs } \\
\hline Total bilirubin, $\mathrm{mg} / \mathrm{dL}$ & $0.6 \pm 0.3$ & $0.5 \pm 0.3$ & 0.13 \\
\hline ALT, U/L & $24.3 \pm 10.5$ & $25.6 \pm 11.3$ & 0.12 \\
\hline AST, U/L & $23.2 \pm 6.6$ & $24.0 \pm 6.9$ & 0.11 \\
\hline
\end{tabular}

Acet, Intravenous acetaminophen; $A S D$, absolute standardized difference; $B M I$, body mass index; $C A B G$, coronary artery bypass graft surgery; $C P B$, cardiopulmonary bypass; $A L T$, alanine aminotransferase; AST, aspartate aminotransferase. *ASD, defined as the absolute difference in means or proportions divided by the pooled standard deviation. Any variables with ASD $>0.20$ were considered imbalanced and were adjusted for in all analyses. †Other surgeries include atrial septal defect or patent foramen ovale closure, left atrial appendage ligation, Maze surgery, pulmonary vein isolation, right atrial or tricuspid valve mass excision, aortic root repair or aortoplasty, pacemaker wire removal, and coronary fistula repair.

\section{Secondary Analyses}

IV acetaminophen had no significant effect on PONV, with an estimated relative risk $(95 \%$ CI $)$ of 0.76 ([0.34-1.68]; $P=.35$, Table 3). Approximately one third of RASS evaluations were not performed at the specified time points; no association between IV acetaminophen and RASS score was found among the available evaluations (Table 3). We originally planned to assess the effect of IV acetaminophen on respiratory depression, but no patients had respiratory depression events.

There was no significant effect of IV acetaminophen on duration of mechanical ventilation, ICU, or hospital LOS, with estimated ratio of geometric means $(95 \%$ adjusted CI) of 1.3 ([0.5-3.2]; $P=.46)$ for duration of mechanical ventilation, 0.9 ([0.7-1.2]; $P=.38$ ) for ICU LOS, and 1.1 ([0.9-1.2]; $P=.12$ ) for hospital LOS (Table 3). IV acetaminophen did not have a significant effect on mean postoperative liver enzyme levels, with estimated ratio of geometric means (95\% adjusted CI) of 1.1 ([0.9-1.2]; $P=.31)$ for ALT, $1.0([0.8-1.2] ; P=.98)$ for AST, and 1.1 ([0.9-1.3]; $P=.40$ ) for total bilirubin (Table 3).

Of note, $73 \%$ of IV acetaminophen and $82 \%$ of placebo patients had fentanyl PCA, and $23 \%$ of IV acetaminophen and $24 \%$ of placebo patients had hydromorphone PCA. A total of $5.5 \%$ of IV acetaminophen and $13.5 \%$ of placebo patients received IV meperidine for shivering, and $27 \%$ of IV acetaminophen and $30 \%$ of placebo patients received oral oxycodone. All opioids were converted to morphine equivalents to account for all given opioids.

\section{DISCUSSION}

We used joint hypothesis testing to evaluate pain intensity and opioid consumption, both of which were likely to be improved by an effective analgesic. We thus planned to conclude that IV acetaminophen is superior to placebo on pain management only if it was noninferior on both outcomes and superior on at least one. This technique allows for a straightforward interpretation of the results while preserving Type I error. ${ }^{20}$ Our results demonstrate that IV acetaminophen was noninferior on
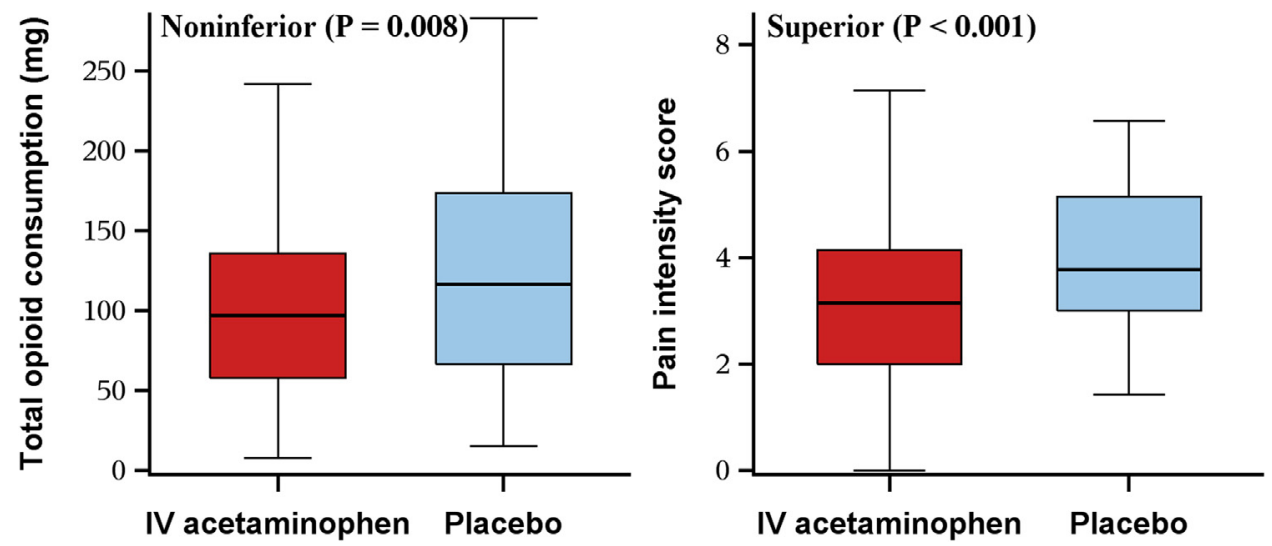

FIGURE 2. IV acetaminophen was noninferior on opioid consumption and superior on pain score. The left panel illustrates the distribution of opioid consumption by treatment group, defined as the total amount of opioids consumed within the first 24 hours after surgery and converted to morphine equivalents $(\mathrm{mg})$. The right panel presents the distribution of pain intensity scores within the first 24 hours by treatment group. Pain intensity scores are based on a $0-10$ Numeric Rating Scale, where 0 is no pain and 10 is the worst possible pain. IV, Intravenous. 
TABLE 2. Effect of IV acetaminophen (vs placebo) on pain management: Total opioid consumption and pain intensity scores within the first 24 hours after surgery

\begin{tabular}{|c|c|c|c|c|c|c|c|c|c|}
\hline Outcome & $\mathbf{n}$ & $\begin{array}{c}\text { Acet } \\
(\mathbf{n}=\mathbf{7 3})\end{array}$ & $\mathbf{n}$ & $\begin{array}{l}\text { Placebo } \\
(n=74)\end{array}$ & Test & Delta & CL* & $\begin{array}{c}\text { Ratio of means } \dagger \\
\text { (CI)* } \\
\text { Acet/placebo }\end{array}$ & $P$ value \\
\hline Opioids (morphine equivalent), mg & 73 & $97[58,136]$ & 74 & $117[66,174]$ & $\begin{array}{l}\text { NI } \\
\text { SUP }\end{array}$ & $\begin{array}{l}1.15 \\
1\end{array}$ & $\begin{array}{l}90 \% \\
95 \%\end{array}$ & $\begin{array}{l}0.89(0.75,1.06) \S \\
0.89(0.73,1.10) \\
\text { Difference in means } \\
\quad(\mathbf{C I})^{*} \\
\quad \text { Acet }- \text { placebo }\end{array}$ & $\begin{array}{l}.008 \\
.28\end{array}$ \\
\hline Pain intensity scores, overall & 73 & $3.1 \pm 1.6 \Upsilon$ & 74 & $4.0 \pm 1.4 \Phi$ & $\begin{array}{l}\text { NI } \\
\text { SUP }\end{array}$ & $\begin{array}{l}1 \\
0\end{array}$ & $\begin{array}{l}90 \% \\
95 \%\end{array}$ & $\begin{array}{l}-0.90(-1.31,-0.50) \S \\
-0.90(-1.39,-0.42) \|\end{array}$ & $\begin{array}{l}<.001 \\
<.001\end{array}$ \\
\hline $4 \mathrm{~h}$ & 59 & $4.6 \pm 2.9$ & 62 & $5.0 \pm 3.1$ & & & & & \\
\hline $6 \mathrm{~h}$ & 63 & $3.5 \pm 2.4$ & 66 & $4.4 \pm 2.4$ & & & & & \\
\hline $8 \mathrm{~h}$ & 64 & $3.2 \pm 2.6$ & 71 & $4.4 \pm 2.3$ & & & & & \\
\hline $12 \mathrm{~h}$ & 71 & $2.4 \pm 2.5$ & 72 & $3.5 \pm 2.6$ & & & & & \\
\hline $16 \mathrm{~h}$ & 72 & $3.0 \pm 2.4$ & 72 & $3.6 \pm 2.4$ & & & & & \\
\hline $20 \mathrm{~h}$ & 72 & $2.9 \pm 2.1$ & 69 & $4.2 \pm 2.0$ & & & & & \\
\hline $24 \mathrm{~h}$ & 72 & $2.5 \pm 2.1$ & & $3.5 \pm 2.0$ & & & & & \\
\hline
\end{tabular}

Summary statistics are reported as mean $\pm \mathrm{SD}$ or median [first quartile, third quartile], as appropriate. Acet, Intravenous acetaminophen; $C L$, confidence level; $C I$, confidence interval; $N I$, noninferior; SUP, superior. *All tests are 1-tailed at the overall .05 significance level. Because both outcomes are required for noninferiority, they were each assessed at the .05 significance level. We performed each superiority test at the .025 significance level because only one significant test was required to reject the null hypothesis (ie, Bonferroni correction). †Ratio of geometric means estimated as exponentiated treatment effect parameter from a multivariable linear regression on log opioid consumption, adjusting for age and diabetes. $\ddagger$ Difference in overall postoperative pain score means based on a repeated measures linear regression model with an autoregressive correlation structure, adjusting for age, time, and diabetes. There was no significant treatment-by time interaction $(P=.35)$. §Noninferiority claimed because the upper confidence limit for the $90 \% \mathrm{CI}$ is less than the specified delta. ||Superiority claimed because the upper confidence limit for the $95 \% \mathrm{CI}$ is less than the specified delta. 9 Overall mean $\pm \mathrm{SD}$ of mean pain score per patient.

opioid consumption and superior on analgesia. Cardiac surgery performed via a median sternotomy is associated with moderate-to-severe pain intensity. Although $1 \mathrm{~g}$ of IV acetaminophen has an analgesic efficacy comparable with $10 \mathrm{mg}$ of morphine ${ }^{21}$ or $30 \mathrm{mg}$ of ketorolac, ${ }^{22}$ it is

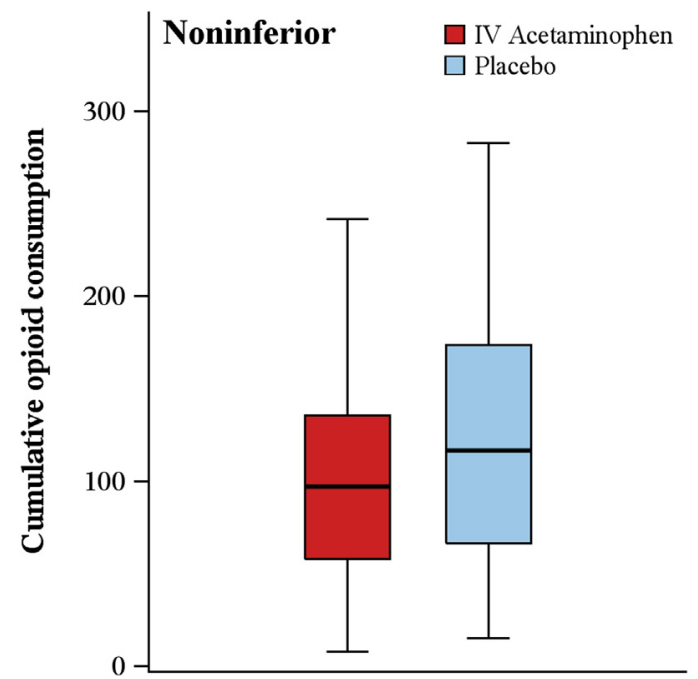

FIGURE 3. Distribution of cumulative opioid consumption by treatment group. Cumulative opioid consumption is defined as the total amount of opioids consumed within the first 24 hours after surgery, converted to morphine equivalents (mg). Boxes represent the first quartile, median, and third quartile whereas whiskers represent the most extreme observations within 1.5 times the interquartile range of the first and third quartiles, respectively. $I V$, Intravenous. unlikely to itself provide sufficient analgesia after cardiac surgery. ${ }^{23}$ Consistent with this theory, IV acetaminophen provided significant analgesia; however, the effect was small, averaging only about 1 point on a 0-10 Numeric Rating Scale.

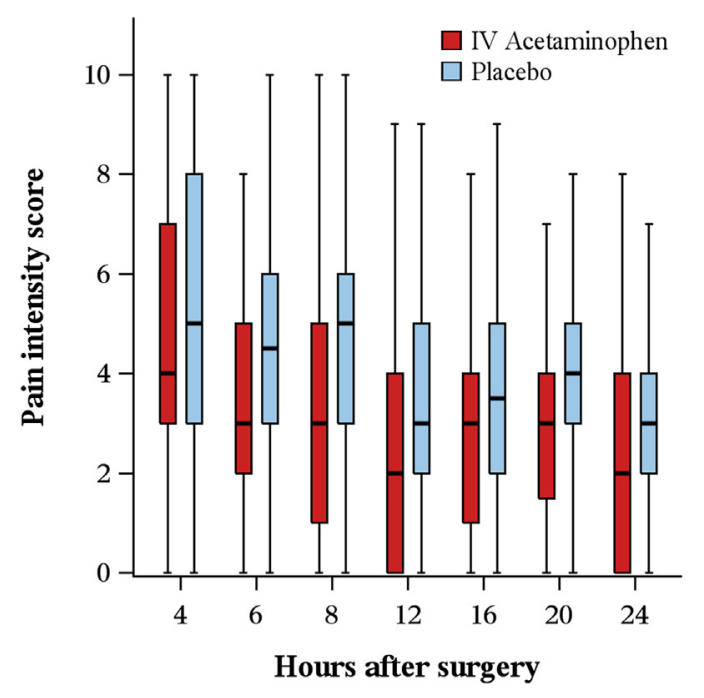

FIGURE 4. Distribution of pain intensity scores over time by treatment group. Pain intensity scores are based on a 0-10 Numeric Rating Scale, where 0 is no pain and 10 is the worst possible pain. Boxes represent the first quartile, median, and third quartile whereas whiskers represent the most extreme observations within 1.5 times the interquartile range of the first and third quartiles, respectively. $I V$, Intravenous. 
Ratio of means of opioid consumption (CI)

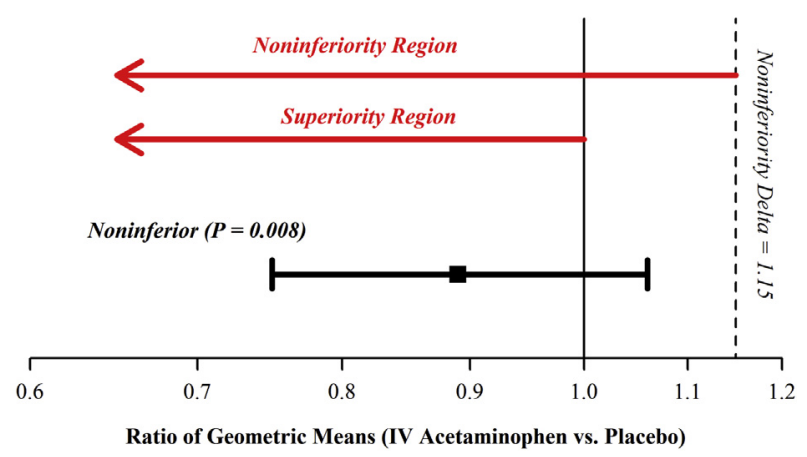

Difference in pain score (CI)

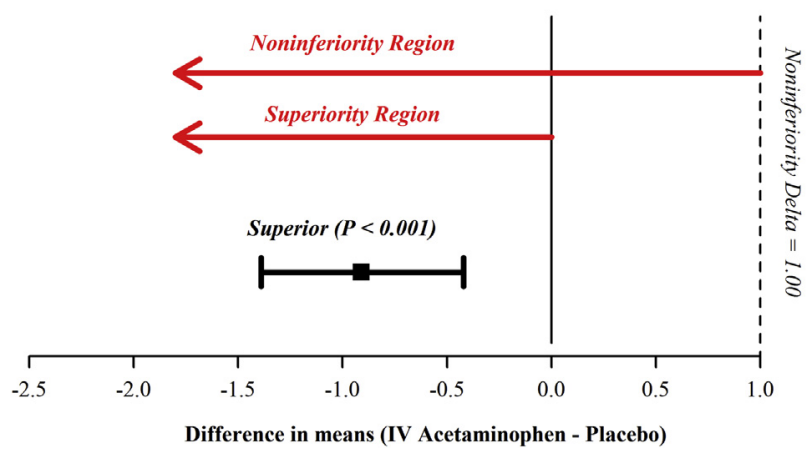

FIGURE 5. Joint hypothesis test results of IV acetaminophen versus placebo on pain management. The effect of IV acetaminophen on opioid consumption was based on the ratio of geometric means estimated from a multivariable linear regression of log opioid consumption. Difference in overall postoperative pain score was based on a repeated measures linear regression model with an autoregressive covariance structure. IV acetaminophen was noninferior $(P=.008)$ but not superior $(P=.28)$ on opioid consumption and both noninferior $(P<.001)$ and superior $(P<.001)$ on pain intensity scores. $C I$, Confidence interval; $I V$, intravenous.

Our results are consistent with those reported by Cattabriga et al, ${ }^{14}$ who reported a significant reduction in pain but not cumulative opioid consumption after cardiac surgery; however, they used a nonstandard approach to provide background analgesia to all their patients by using a fixed dose of both loading and continuous infusion of IV tramadol, which is unavailable in the United States. Certainly, improved analgesia in patients treated with a tramadol-based background analgesia might not be generalizable to our patient population. Also, the use of opioids only as a rescue analgesic limited their ability to evaluate opioid consumption as a primary end point. To be able to do so, they would have needed to increase their sample size by almost 5-fold. In contrast, we used opioids for both rescue and background analgesia which allowed us to use a joint hypothesis testing to evaluate both pain intensity scores and opioid consumption as our primary endpoint.
IV acetaminophen has been reported to reduce pain more consistently than reducing opioid consumption. ${ }^{24}$ Indeed, other trials also report improved pain scores without a reduction in opioid consumption. ${ }^{12,14,25}$ Our study was designed to identify a 30\% difference in opioid consumption between the acetaminophen and placebo groups. Opioid consumption was an estimated 11\% lower $(95 \%$ CI $27 \%$ decrease to $10 \%$ increase) with IV acetaminophen, yielding a conclusion of noninferiority versus placebo because the upper limit is less than the $15 \%$ a priori defined noninferiority delta. We had $90 \%$ power to detect superiority, given a true reduction of $30 \%$ or more in mean opioid consumption, but our results do not suggest a benefit nearly that large.

We found that addition of IV acetaminophen to an opioid-based analgesic regimen had no significant effect on PONV, RASS scores, duration of mechanical ventilation, or ICU and hospital LOS. It was unsurprising to find comparable opioid-related adverse effects, given that opioid consumption also was similar in each treatment group. Only few studies showed significant reduction of opioid-related adverse effects. $11,12,26,27$ The reduction of PONV in 1 study correlated with the reduction of pain but not with reduction in opioid consumption, suggesting that reduced PONV was mediated mainly through more effective analgesia. ${ }^{27}$ Our study was underpowered to detect such reduction.

Consistent with our findings, 2 large recent systematic reviews and meta-analyses did not report a reduction in opioid-related PONV or other opioid-related adverse effects with IV acetaminophen, even though they reported a $20 \%-30 \%$ reduction in opioid consumption. ${ }^{28,29}$ More reduction in opioid consumption is probably required to effect a consistent reduction in opioid-related adverse effects. ${ }^{30}$ Alternatively, this lack of reduction in adverse effects might occur because many of those effects are reinforced by neural reflexes triggered by surgical stress; simply reducing opioid consumption might thus be insufficient to comparably reduce opioid related adverse effects. $^{31}$

IV acetaminophen reduces initial hepatic exposure and avoids first-pass hepatic metabolism; the IV preparation is thus assumed to be safer than oral acetaminophen from a hepatic perspective. ${ }^{32}$ Daily administration of $4 \mathrm{~g}$ of acetaminophen significantly increases ALT plasma concentrations in healthy volunteers ${ }^{33}$; however, increases in liver enzymes were not reported in studies that evaluated the safety of a short-term regimen of $4 \mathrm{~g}$ of IV acetaminophen daily in both hospitalized patients with a mix of surgical and medical patients and in cardiac critical care units. ${ }^{34,35}$ In our study, there was no increase in liver enzymes in patients who received IV acetaminophen compared with the placebo group.

This study had few limitations. IV fentanyl was the default postoperative opioid, but patients were permitted to receive 
TABLE 3. Effect of IV acetaminophen (vs placebo) on secondary outcomes within the first 24 hours after surgery

\begin{tabular}{|c|c|c|c|c|c|c|}
\hline Secondary outcome & $\mathbf{n}$ & $\begin{array}{c}\text { Acet } \\
(n=73)\end{array}$ & $\mathbf{n}$ & $\begin{array}{l}\text { Placebo } \\
(n=74)\end{array}$ & $\begin{array}{c}\text { Relative risk* } \text { (CI }) \dagger^{\dagger} \\
\text { Acet vs placebo }\end{array}$ & $P$ value \\
\hline PONV, n (\%) & 73 & $16(22)$ & 74 & $21(28)$ & $\begin{array}{c}0.76(0.34-1.7) \\
\text { Difference in medians } \ddagger(\mathrm{CI}) \dagger \\
\text { Acet }- \text { placebo }\end{array}$ & .35 \\
\hline \multicolumn{7}{|l|}{ RASS score } \\
\hline $\begin{array}{l}8 \mathrm{~h} \\
16 \mathrm{~h} \\
24 \mathrm{~h}\end{array}$ & $\begin{array}{l}48 \\
39 \\
39\end{array}$ & $\begin{array}{l}0[-1,0] \\
0[-1,0] \\
0[0,0]\end{array}$ & $\begin{array}{l}41 \\
43 \\
31\end{array}$ & $\begin{array}{l}0[-1,0] \\
0[0,0] \\
0[0,0]\end{array}$ & $\begin{array}{c}0(0,0) \\
0(0,0) \\
0(0,0) \\
\text { Ratio of means } \S(\mathrm{CI}) \dagger \\
\text { Acet/placebo }\end{array}$ & $\begin{array}{l}.13 \\
.44 \\
.38\end{array}$ \\
\hline Duration of initial mechanical ventilation, min & 73 & $214[143,345]$ & 74 & $190[139,381]$ & $1.3(0.52-3.2)$ & .46 \\
\hline ICU LOS, h & 73 & $27[25,42]$ & 74 & $26[24,48]$ & $0.93(0.74-1.2)$ & .38 \\
\hline Hospital LOS, d & 73 & $6.2[5.3,7.3]$ & 74 & $6.1[5.1,7.2]$ & $1.1(0.94-1.2)$ & .12 \\
\hline ALT (U/L), overall & 73 & $18[15,23]$ & 74 & $19[15,24] \|$ & $1.1(0.90-1.2)$ & .31 \\
\hline POD 1 & 73 & $18[16,24]$ & 74 & $19[15,25]$ & & \\
\hline POD 2 & 73 & $17[14,22]$ & 74 & $18[15,23]$ & & \\
\hline AST (U/L), overall & 73 & $35[30,45] \|$ & 74 & $33[29,51] \|$ & $1.0(0.80-1.2)$ & .98 \\
\hline POD 1 & 73 & $40[32,54]$ & 74 & $40[32,57]$ & & \\
\hline POD 2 & 73 & $31[24,42]$ & 74 & $31[23,45]$ & & \\
\hline Total bilirubin (mg/dL), overall & 73 & $0.7[0.5,0.9] \|$ & 74 & $0.7[0.5,0.8] \|$ & $1.1(0.87-1.3)$ & .40 \\
\hline POD 1 & 73 & $0.7[0.5,1.0]$ & 74 & $0.7[0.5,0.8]$ & & \\
\hline POD 2 & 73 & $0.6[0.4,0.8]$ & 74 & $0.6[0.4,0.8]$ & & \\
\hline
\end{tabular}

Summary statistics are reported as n (\%) or median [first quartile, third quartile], as appropriate. Acet, Intravenous acetaminophen; $C I$, confidence interval; $P O N V$, postoperative nausea and vomiting; RASS, Richmond Agitation Sedation Scale; $I C U$, intensive care unit; $L O S$, length of stay; $A L T$, alanine aminotransferase; $P O D$, postoperative day; $A S T$, aspartate aminotransferase. *Relative risk of PONV in Acet versus placebo patients estimated from a multivariable logistic regression model using the log link and adjusting for age and diabetes. $\nmid$ Significance criterion of 0.006 used for each secondary outcome (ie, Bonferroni correction, $0.05 / 8$ ). Groups were compared at the 0.002 significance level for each RASS assessment (ie, 0.006/3). ‡Difference in medians of IV acetaminophen versus placebo patients based on Wilcoxon rank sum test and Hodges-Lehmann estimation of location shift. §Secondary outcomes were log-transformed to meet model assumptions. The treatment effect on duration of mechanical ventilation, ICU LOS, and hospital LOS was assessed by use of the ratio of geometric means from separate multivariable logistic regression models, each adjusting for age and diabetes. Using an autoregressive correlation structure and adjusting for age, diabetes, and time of measurement, we used a similar repeated-measures model to assess the effect of Acet on liver enzymes. $\|$ Median [first quartile, third quartile] of mean liver enzyme per patient.

IV hydromorphone, meperidine, or oral oxycodone if clinically indicated. We used standard conversions among the allowed opioids, but all conversion systems are only rough approximations, especially given highly variable context-sensitive half-lives of various opioids. Occasional pain scores were missing, mostly in patients who remain intubated; some patients thus contributed fewer pain scores than others to our mixed-effects model. The amount and pattern of missing data, however, were similar in the randomized groups making it unlikely that missing data much altered our results. In addition, about a third of the RASS scores-a secondary outcome-were missing. Our analyses assume that data were missing at random. This study is underpowered to make conclusions about secondary endpoints, so secondary results should be interpreted with caution.

In conclusion, IV acetaminophen significantly reduced pain intensity scores by about 1 point on a $0-10$ scale but did not significantly reduce opioid consumption after cardiac surgery. IV acetaminophen alone, unsurprisingly, provided insufficient analgesia for patients recovering from median sternotomy. It may be used, however, as an effective component of a multimodal analgesic strategy after cardiac surgery.

\section{Conflict of Interest Statement}

Authors have nothing to disclose with regard to commercial support.

\section{References}

1. Crews JC. Multimodal pain management strategies for office-based and ambulatory procedures. JAMA. 2002;288:629-32.

2. American Society of Anesthesiologists Task Force on Acute Pain Management. Practice guidelines for acute pain management in the perioperative setting: An updated report by the American Society of Anesthesiologists Task Force on Acute Pain Management. Anesthesiology. 2012;116:248-73.

3. Sachs CJ. Oral analgesics for acute nonspecific pain. Am Fam Physician. 2005; 71:913-8.

4. Jibril F, Sharaby S, Mohamed A, Wilby KJ. Intravenous versus oral acetaminophen for pain: Systematic review of current evidence to support clinical decisionmaking. Can J Hosp Pharm. 2015;68:238-47.

5. Moller PL, Sindet-Pedersen S, Petersen CT, Juhl GI, Dillenschneider A, Skoglund LA. Onset of acetaminophen analgesia: comparison of oral and intravenous routes after third molar surgery. Br J Anaesth. 2005;94:642-8.

6. Brett CN, Barnett SG, Pearson J. Postoperative plasma paracetamol levels following oral or intravenous paracetamol administration: a double-blind randomised controlled trial. Anaesth Intensive Care. 2012;40:166-71.

7. Singla NK, Parulan C, Samson R, Hutchinson J, Bushnell R, Beja EG, et al. Plasma and cerebrospinal fluid pharmacokinetic parameters after single-dose administration of intravenous, oral, or rectal acetaminophen. Pain Pract. 2012; 12:523-32.

8. Cazacu I, Mogosan C, Loghin F. Safety issues of current analgesics: an update. Clujul Med. 2015;88:128-36. 
9. Duggan ST, Scott LJ. Intravenous paracetamol (acetaminophen). Drugs. 2009; 69:101-13.

10. Sinatra RS, Jahr JS, Reynolds LW, Viscusi ER, Groudine SB, PayenChampenois C. Efficacy and safety of single and repeated administration of 1 gram intravenous acetaminophen injection (paracetamol) for pain management after major orthopedic surgery. Anesthesiology. 2005;102:822-31.

11. Memis D, Inal MT, Kavalci G, Sezer A, Sut N. Intravenous paracetamol reduced the use of opioids, extubation time, and opioid-related adverse effects after major surgery in intensive care unit. J Crit Care. 2010;25:458-62.

12. Cakan T, Inan N, Culhaoglu S, Bakkal K, Basar H. Intravenous paracetamol improves the quality of postoperative analgesia but does not decrease narcotic requirements. J Neurosurg Anesthesiol. 2008;20:169-73.

13. Nishimoto RN. OFIRMEV: An old drug becomes new again. Anesth Prog. 2014; 61:99-102.

14. Cattabriga I, Pacini D, Lamazza G, Talarico F, Di Bartolomeo R, Grillone G, et al. Intravenous paracetamol as adjunctive treatment for postoperative pain after cardiac surgery: a double blind randomized controlled trial. Eur J Cardiothorac Surg. 2007;32:527-31.

15. Breivik H, Borchgrevink PC, Allen SM, Rosseland LA, Romundstad L, Hals EK, et al. Assessment of pain. Br J Anaesth. 2008;101:17-24.

16. Turan A, Atim A, Dalton JE, Keeyapaj W, Chu W, Bernstein E, et al. Preoperative angiotensin-converting enzyme inhibitor use is not associated with increased postoperative pain and opioid use. Clin J Pain. 2013;29:1050-6.

17. Sessler CN, Gosnell MS, Grap MJ, Brophy GM, O’Neal PV, Keane KA, et al. The Richmond Agitation-Sedation Scale: validity and reliability in adult intensive care unit patients. Am J Respir Crit Care Med. 2002;166:1338-44.

18. Cohen J. Statistical Power Analysis for the Behavioral Sciences. 2nd ed. Hillsdale, NJ: Lawrence Erlbaum Associates, Inc.; 1988.

19. Mascha EJ, Sessler DI. Equivalence and noninferiority testing in regression models and repeated-measures designs. Anesth Analg. 2011;112:678-87.

20. Mascha EJ, Turan A. Joint hypothesis testing and gatekeeping procedures for studies with multiple endpoints. Anesth Analg. 2012;114:1304-17.

21. Van Aken H, Thys L, Veekman L, Buerkle H. Assessing analgesia in single and repeated administrations of propacetamol for postoperative pain: comparison with morphine after dental surgery. Anesth Analg. 2004;98:159-65. table of contents.

22. Zhou TJ, Tang J, White PF. Propacetamol versus ketorolac for treatment of acute postoperative pain after total hip or knee replacement. Anesth Analg. 2001;92: 1569-75.

23. Pettersson PH, Jakobsson J, Owall A. Intravenous acetaminophen reduced the use of opioids compared with oral administration after coronary artery bypass grafting. J Cardiothorac Vasc Anesth. 2005;19:306-9.
24. Macario A, Royal MA. A literature review of randomized clinical trials of intravenous acetaminophen (paracetamol) for acute postoperative pain. Pain Pract. 2011;11:290-6.

25. Wininger SJ, Miller H, Minkowitz HS, Royal MA, Ang RY, Breitmeyer JB, et al A randomized, double-blind, placebo-controlled, multicenter, repeat-dose study of two intravenous acetaminophen dosing regimens for the treatment of pain after abdominal laparoscopic surgery. Clin Ther. 2010;32:2348-69.

26. De Oliveira GS Jr, Castro-Alves LJ, McCarthy RJ. Single-dose systemic acet aminophen to prevent postoperative pain: a meta-analysis of randomized controlled trials. Clin J Pain. 2015;31:86-93.

27. Apfel CC, Turan A, Souza K, Pergolizzi J, Hornuss C. Intravenous acetaminophen reduces postoperative nausea and vomiting: a systematic review and meta-analysis. Pain. 2013;154:677-89.

28. McNicol ED, Tzortzopoulou A, Cepeda MS. Single-dose intravenous paracetamol or propacetamol for prevention or treatment of postoperative pain: A systematic review and meta-analysis. Br J Anaesth. 2011;106:764-75.

29. Tzortzopoulou A, McNicol ED, Cepeda MS, Francia MB, Farhat T, Schumann R Single dose intravenous propacetamol or intravenous paracetamol for postoperative pain. Cochrane Database Syst Rev. 2011;CD007126.

30. Marret E, Kurdi O, Zufferey P, Bonnet F. Effects of nonsteroidal antiinflammatory drugs on patient-controlled analgesia morphine side effects: meta-analysis of randomized controlled trials. Anesthesiology. 2005;102: 1249-60.

31. Kehlet H, Werner MU. Role of paracetamol in the acute pain management Drugs. 2003;63(Spec No 2):15-22.

32. Jahr JS, Lee VK. Intravenous acetaminophen. Anesthesiol Clin. 2010;28 619-45.

33. Watkins PB, Kaplowitz N, Slattery JT, Colonese CR, Colucci SV Stewart PW, et al. Aminotransferase elevations in healthy adults receiving 4 grams of acetaminophen daily: a randomized controlled trial. JAMA 2006;296:87-93.

34. Ahlers SJ, Van Gulik L, Van Dongen EP, Bruins P, Tibboel D, Knibbe CA Aminotransferase levels in relation to short-term use of acetaminophen four grams daily in postoperative cardiothoracic patients in the intensive care unit. Anaesth Intensive Care. 2011;39:1056-63.

35. Candiotti KA, Bergese SD, Viscusi ER, Singla SK, Royal MA, Singla NK. Safety of multiple-dose intravenous acetaminophen in adult inpatients. Pain Med. 2010 11:1841-8.

Key Words: intravenous acetaminophen, cardiac surgery, acute pain management, multimodal analgesia 
TABLE E1. Opioid dosage conversion chart $^{16}$

\begin{tabular}{lcc}
\hline & \multicolumn{2}{c}{ Equianalgesic dose } \\
\cline { 2 - 3 } Morphine-like agonists & Oral & Intravenous \\
\hline Morphine & $30 \mathrm{mg}$ & $10 \mathrm{mg}$ \\
Fentanyl & - & $100 \mu \mathrm{g}$ \\
Hydromorphone & $7.5 \mathrm{mg}$ & $1.5 \mathrm{mg}$ \\
Meperidine & $300 \mathrm{mg}$ & $75 \mathrm{mg}$ \\
Oxycodone & $20 \mathrm{mg}$ & - \\
\hline
\end{tabular}

